\title{
BRAKE CONTROL COMBINED WITH PREDICTION TO PREVENT THE ROLLOVER OF HEAVY VEHICLES
}

\author{
P. Gaspar, Z. Szabo, J. Bokor \\ Computer and Automation Research Institute, \\ Hungarian Academy of Sciences, Hungary \\ Kende u. 13-17, H-1111 Budapest, Hungary
}

\begin{abstract}
In this paper an active brake control to decrease the rollover risk of heavy vehicles is proposed. The brake system is activated when the vehicle comes close to rolling over. To enhance the performance of the active brake, this mechanism is extended with a prediction procedure, in which the critical values are predicted in advance using a short time interval. In order to predict an imminent rollover, an observer-based prediction algorithm is proposed to estimate in advance the load transfers. The control design is based on the Linear Parameter Varying (LPV) Model of yaw-roll dynamics of heavy vehicles. In the control design both the performance demands and the model uncertainties are taken into consideration. The control mechanism is demonstrated in a double lane change maneuver. Copyright ${ }^{\circledR} 2005$ IFAC
\end{abstract}

Keywords: vehicle dynamics, linear parameter varying control, nonlinear control systems, active control, stability, robustness

\section{INTRODUCTION}

The aim of rollover prevention is to provide the vehicle with the ability to resist overturning moments generated during vehicle maneuvers. Roll stability is determined by the height of the center of mass, the track width and the kinematic properties of the suspensions. The primary overturning moment arises from the lateral acceleration acting on the center of gravity of the vehicle. More destabilizing moment arises during the cornering manoeuver when the center of gravity of the vehicle shifts laterally. The roll stability of the vehicle can be guaranteed if the sum of the destabilizing moment is compensated during a lateral manoeuver.

Several schemes concerned with the possible active intervention into vehicle dynamics have been proposed. These approaches employ active anti- roll bars, active steering, active braking, active suspensions, or a combination of them, see (Ackermann and Odenthal, 1999; Chen and Peng, 2001; Kim and Park, 2004; Lin et al., 1996; Mammar and Koenig, 2002; Palkovics et al., 1999; Sampson and Cebon, 2003). A combined control structure, in which active anti-roll bars are combined with an active brake control, has also been developed in order to guarantee a fault-tolerant behavior of the controlled system, see (Gaspar et al., 2004; Gaspar et al., 2005).

In this paper the LPV control of the active brake system is developed. The control of the active brake system must guarantee the performance demands of the vehicle and take the uncertainty of the model into consideration. The operation of the active brake is the following. When a rollover is imminent and this dangerous situation persists, the active brake system must generate 
unilateral brake forces in order to reduce the rollover risk. Thus, the brake is activated only in critical situations, i.e. when the vehicle comes close the rolling over. In this paper the detection of an imminent rollover is based on the monitoring of the lateral load transfers for both axles. If the lateral load transfer takes on a critical value, the brake is activated.

The problem with this solution is that when a rollover is imminent, the switching of the brake can cause a dangerous slip of the vehicle. This problem can be solved by decreasing the critical value, in which the brake is activated, however, in this case the activation of the brake will be more frequent and the duration of the active braking will be longer.

In this paper the LPV control of the active brake system is extended with a new prediction procedure, in which the critical values are predicted in advance using a short time interval. That is, the values of the lateral load transfer are predicted in order to determine whether these values may exceed the critical value corresponding to the rollover. If in this short time interval the predicted values exceed the critical lateral load transfer, the brake is activated. The result is that in the predicted case the magnitude and the duration of the braking force required usually is smaller than in the case without prediction. The active brake extended with prediction procedure improves the efficiency of the braking.

The structure of the paper is as follows. In Section 2 the yaw-roll model in which the forward velocity changes in time is constructed. Section 3 presents the solution of the observer based prediction. Section 4 presents the control design based on the LPV method. Section 5 demonstrates the results of the control design. Finally, Section 6 contains some concluding remarks.

\section{AN LPV MODEL OF THE YAW-ROLL DYNAMICS}

Figure 1 illustrates the combined yaw-roll dynamics of the vehicle, which is modelled by a threebody system, in which $m_{s}$ is the sprung mass, $m_{u, f}$ is the unsprung mass at the front including the front wheels and axle, and $m_{u, r}$ is the unsprung mass at the rear. The signals are the lateral acceleration $a_{y}$, the side slip angle of the sprung mass $\beta$, the heading angle $\psi$, the yaw rate $\dot{\psi}$, the roll angle of the sprung mass $\phi$, the roll rate $\dot{\phi}$, the roll angle of the unsprung mass at the front axle $\phi_{t, f}$ and at the rear axle $\phi_{t, r} . \delta_{f}$ is the front wheel steering angle. $v$ is the forward velocity. The total axle loads are $F_{z l}$ and $F_{z r} . v$ is the forward velocity.
The equations of the yaw-roll dynamics can be expressed in the state space representation form:

$$
\begin{aligned}
& \dot{x}(t)=A(\rho) x(t)+B_{1}(\rho) d(t)+B_{2}(\rho) u(t) \\
& y(t)=C x(t)
\end{aligned}
$$

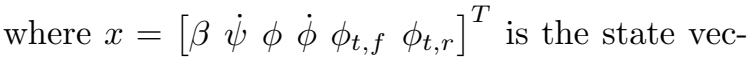
tor. In equation (1) the system matrices depend on the forward velocity of the vehicle nonlinearly. If $v$ is chosen as a scheduling parameter, an LPV model of the yaw-roll dynamics is defined: $\rho=v$. The disturbance signal is the front wheel steering angle, $d(t)=\delta_{f}(t)$.

The control input is the difference of brake forces between the left and the right hand side of the vehicle $u(t)=\Delta F_{b}(t)$. The control input provided by the brake system generates a yaw moment, which affects the lateral tire forces directly. In our case it is assumed that the brake force difference $\Delta F_{b}$ provided by the controller is applied to the rear axle: $\Delta F_{b}=F_{b, r l}-F_{b, r r}$. This assumption does not restrict the implementation of the controller because it is possible that the control action be distributed on the front and the rear wheels on either the two sides. The reason for distributing the control force between the front and rear wheels is to minimize the wear of the tires. In this case a logic is required which calculates the brake forces for the wheels.

The measured output $y(t)$ contains the yaw rate $\dot{\psi}$, the roll rate $\dot{\phi}$ and the perturbed lateral acceleration of the sprung mass $a_{y}$ where the lateral acceleration is as follows: $a_{y}=v \dot{\beta}+v \dot{\Psi}-h \ddot{\Phi}$.

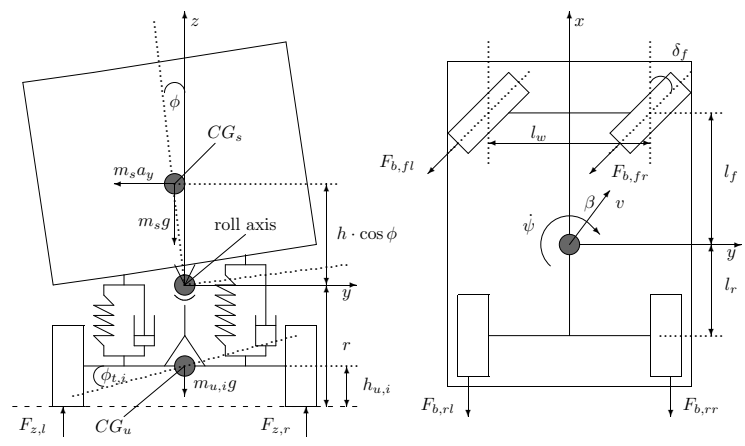

Fig. 1. Illustration of the vehicle model

\section{OBSERVER BASED PREDICTION OF THE LATERAL LOAD TRANSFERS}

Three moments act on the vehicle: the overturning moment arising from the lateral acceleration $\left(m a_{y} h\right)$, the restoring moment arising from the lateral load transfer from the inside tires onto the outside tires $\left(\Delta F_{z} \ell_{w}\right)$, and the lateral displacement moment arising from the roll motion, which displaces the center of mass laterally from the nominal center line $(m g h \Phi)$. Roll stability 
is achieved by limiting the lateral load transfers for both axles, $\Delta F_{z, l}$ and $\Delta F_{z, r}$, to below the levels for wheel lift-off. The lateral load transfer is calculated:

$$
\Delta F_{z, i}=\frac{k_{t, i} \phi_{t, i}}{l_{w}}
$$

where $k_{t, i}$ is the stiffness of the tires at the front and rear axles, $\phi_{t, i}$ is the roll angle of the unsprung mass and $l_{w}$ is the vehicle's width, and $i=f, r$ denotes the front and rear of the vehicle.

The tire contact force is guaranteed if $\frac{m g}{2} \pm \Delta F_{z}>$ 0 for both sides of the vehicle. This requirement leads to the definition of the normalized load transfer, which is the ratio of the lateral load transfers at the front and rear axles:

$$
R_{i}=\frac{\Delta F_{z, i}}{m_{i} g} .
$$

where $m_{i}$ is the mass of the vehicle in the front and the rear. The normalized load transfer $R_{i}$ value corresponds to the largest possible load transfer. If the $R_{i}$ takes on the value \pm 1 then the inner wheels in the bend lift off. The limit cornering condition occurs when the load on the inside wheels has dropped to zero and all the load has been transferred onto the outside wheels.

Using the brake system of the vehicle a yaw moment can be generated by unilateral brake forces, which can reduce the lateral acceleration directly.

In this paper the detection of an imminent rollover is based on the monitoring of the lateral load transfers for both axles. In the control design the predicted value of the normalized load transfer is used. It is generated in two steps:

Step 1 : The design of an observer to generate $\tilde{R}$ based on the measured signals, $a_{y}, \dot{\psi}$ and $\dot{\phi}$, where $a_{y}$ is the lateral acceleration.

Step 2 : Prediction in a short period $\hat{R}$ based on the observed $\tilde{R}$.

It can be shown that for the LPV model of the yaw-roll dynamics the state matrices depend affinely on the parameter vector. This class of systems can be described as:

$$
\begin{aligned}
\dot{x}(t) & =A(\rho) x(t)+B_{2}(\rho) u(t) \\
y(t) & =C x(t),
\end{aligned}
$$

where $A(\rho)=A_{0}+\rho_{1} A_{1}+\cdots+\rho_{N} A_{N}, B_{2}(\rho)=$ $B_{0}+\rho_{1} B_{1}+\cdots+\rho_{N} B_{N}$ and $\rho_{i}$ are time varying parameters. It is assumed that each parameter $\rho_{i}$ and its derivatives $\dot{\rho}_{i}$ range between known extremal values $\rho_{i}(t) \in\left[-\bar{\rho}_{i}, \bar{\rho}_{i}\right]$ and $\dot{\rho}_{i}(t) \in$ $\left[-\bar{\rho}_{i}, \bar{\rho}_{i}\right]$, respectively. Let us denote this parameter set by $\mathcal{P}$. For the model of the yaw-roll dynamics $N=2$ and the parameters are $\rho_{1}=v$ and $\rho_{2}=\frac{1}{v}$.
In order to make a prediction for the lateral load transfers must be estimated the roll angles of the unsprung masses $\phi_{t, i}$. It was proposed to design a Luenberger type observer for the system. The form of the observer is the following:

$$
\dot{\eta}=(A(\rho)+K(\rho) C) \eta+B(\rho) u-K(\rho) y .
$$

Concerning the question of stability, let us recall that an LPV system is said to be quadratically stable if there exist a matrix $P=P^{T}>0$ such that

$$
A(\rho)^{T} P+P A(\rho)<0
$$

for all the parameters $\rho \in \mathcal{P}$. A necessary and sufficient condition for a system to be quadratically stable is that the condition in equation (8) holds for all the corner points of the parameter space, i.e., one can obtain a finite system of LMI's that has to be fulfilled for $A(\rho)$ with a suitable positive definite matrix $P$, see (Gahinet et al., 1996; Wu et al., 1996).

In order to obtain a quadratically stable observer the LMI:

$$
(A(\rho)+K(\rho) C)^{T} P+P(A(\rho)+K(\rho) C)<0
$$

must hold for suitable $G(\rho)$ and $P=P^{T}>0$. By introducing the auxiliary variable $G(\rho)=P K(\rho)$, one has to solve the following set of LMIs on the corner points of the parameter space:

$$
A(\rho)^{T} P+P A(\rho)+C^{T} K(\rho)^{T}+K(\rho) C<0 .
$$

By solving these LMIs one can obtain a suitable observer gain. The obtained observer give us an estimation for the state vector, hence, an estimation for the values of the the roll angles of the unsprung masses $\phi_{t, i}$. With these values the valuers of the normalized load transfers are calculated $\tilde{R}_{i}$. These values will be considered as input data for the prediction step.

Since the inputs of the model (1) are not known in advance it is considered to be more reliable to use a simple linear model, e.g., an AR model structure, to model only the evolution of the normalized load transfer based on measurements of a given length. Therefore the prediction algorithm fits an AR model structure of order $n$ to the sampled values of length $L_{w}$ and then using this model it predicts the values of $\hat{R}_{i}$ for a horizon of $\ell_{h}$ samples. For the implementation a recursive least squares estimation method is used, see (Ljung, 1987).

Based on the recursive algorithm $\hat{R}$ is estimated. The prediction is based on values taken within a short time interval. The model order is set at $n=2, \ell_{w}$ consists of 50 sampling points and $\ell_{h}$ is chosen to be 5 sampling points. The forgetting 
factor $\lambda$ in the recursive AR algorithm is set at 0,97 . The value of $\ell_{h}$ provides a prediction interval between $0.5 \mathrm{~m}$ and $2 \mathrm{~m}$ depending on the forward velocity. In the control design step the predicted values of the normalized load transfer $\hat{R}$ are applied instead of the value $R$ at the time $t_{i}$. Here $\hat{R}$ is the predicted value at the time $t_{i+\ell_{h}}$.

\section{CONTROL DESIGN BASED ON THE LPV METHOD}

Consider the closed-loop system in Figure 2, which includes the feedback structure of the model $G(\rho)$ and controller $K(\rho)$, and elements associated with the uncertainty models and performance objectives. In the diagram, $z$ represents the performance outputs, i.e. the lateral acceleration, the lateral load transfers and the brake forces. $n$ is the measurement noise, and $e$ and $d$ are the input and output of the uncertainty block.

The weighting function $W_{p}$ represents the weighting function for performance outputs, and it contains the components $W_{p_{a}}$ and $W_{p_{F_{b}}}$. The purpose of the weighting functions is to keep the lateral acceleration, the lateral load transfers and the control inputs small over the desired frequency range. That is, weights should be large in a frequency range where small signals are desired and small where larger performance outputs can be tolerated. The weighting function $W_{p_{a}}$ is selected as:

$$
W_{p_{a}}=\phi_{a} \frac{(s / 2000+1)}{(s / 12+1)}
$$

Here, it is assumed that in the low frequency domain the steering angle at the lateral accelerations of the body should be rejected by a factor of $\phi_{a}$. To avoid the actuator saturation the weight $W_{p_{F_{b}}}$ applied for the brake force is $1 / 10$.

The $\phi_{a}$ is chosen to be parameter-dependent, i.e., the function of the predicted values of the normalized load transfer $\hat{R}$. The parameter dependent gain $\phi_{a}$ captures the relative importance of the acceleration response. When $\hat{R}$ is small, i.e., when the vehicle is not in an emergency, $\phi_{a}(R)$ is small, indicating that the LPV controller should not focus on minimizing acceleration. On the other hand, when $\hat{\hat{R}}$ approaches the critical value, i.e. $\phi_{a}(\hat{R})$ is large, indicating that the controller should focus on preventing the rollover. In this paper the parameter dependence of the gain is characterized by the constants $R_{1}$ and $R_{2} . R_{1}$ defines the critical status when the vehicle is close to rolling over. The closer $R_{1}$ is to 1 , the later the controller will be activated. $R_{2}$ parameter shows how fast the controller should focus on minimizing the lateral acceleration. The parameter dependent gain $\phi_{a}(\hat{R})$ in equation $(11)$ is as follows:

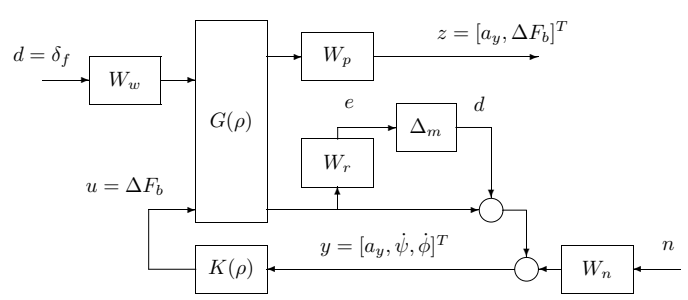

Fig. 2. The closed-loop interconnection structure

$$
\phi_{a}(\hat{R})=\left\{\begin{array}{cl}
0 & \text { if } \quad|\hat{R}|<R_{1} \\
\frac{2}{R_{2}-R_{1}}\left(|\hat{R}|-R_{1}\right) & \text { if } \quad R_{1} \leq|\hat{R}| \leq R_{2} \\
2 & \text { otherwise }
\end{array}\right.
$$

The uncertainties of the model are represented by $W_{r}$ and $\Delta_{m}$. Design models used for roll stability control typically exhibit high fidelity at lower frequencies $(\omega<10 \mathrm{~Hz})$, but they deteriorate at higher frequencies due to neglected effects. Thus, $W_{r}$ is selected as $W_{r}=2.25 \frac{s+20}{s+450}$. The input scaling weight $W_{\delta}$ normalizes the steering angle to the maximum expected command. It is selected as $5 \pi / 180$, which corresponds to 5 degrees of steering angle command. $W_{n}$ is selected as a diagonal matrix, which accounts for sensor noise models in the control design. The noise weight for the lateral acceleration is chosen $0.01 \mathrm{~m} / \mathrm{s}^{2}$ and both for the yaw rate and the roll rate is $0.01 \mathrm{deg} / \mathrm{sec}$.

In the LPV model of the yaw-roll motion two parameters are selected. The forward velocity $v$ is a measured signal. The prediction of the normalized lateral load transfer at the rear side $\hat{R}$ can be calculated through the observer design and the prediction algorithm.

The LPV synthesis used in this research requires a gridded parameter space. For the interconnection structure, $\mathcal{H}_{\infty}$ controllers are synthesized for several values of velocity in a range $v=$ $[30 \mathrm{kmh}, 100 \mathrm{kmh}]$. The spacing of the grid points is selected on the basis of how well the $\mathcal{H}_{\infty}$ point designs perform for plants around the design point. 8 grid points are selected for the scheduling parameter design space. The rear load transfer parameter space is also grided as $R=[0,0.5,0.85,0.95,1]$. Weighting functions for both the performance and robustness specifications are defined in all of the grid points. With respect to the robustness requirement, the same frequency weighting functions are applied in the entire parameter space and the effect of the scheduling parameter is neglected. It is a reasonable engineering assumption, since the uncertainty, i.e. unmodelled dynamics and parametric uncertainties, does not depend on the forward velocity. 


\section{SIMULATION RESULTS}

In this section, the operation of the active brake, which is based on an LPV control, is illustrated in a a double lane change maneuver. The maneuver has a $2 \mathrm{~m}$ path deviation over $100 \mathrm{~m}$. The size of the path deviation is chosen to test a real obstacle avoidance emergency. The steering angle input is generated in such a way that the vehicle with a passive brake control comes close to rolling over during the maneuver and its normalized load transfers are over \pm 1 . The vehicle velocity is 70 $k p h$.

In the first example, the active brake is compared with a passive system. In case of the passive brake mechanism the brake force is only provided by the driver and it is distributed equally to all of the wheels and they do not generate yaw moment around the center of mass. Figure 3 shows solid (active brake) and dashed (passive) lines the time responses to the double lane change steering input, i.e. $a_{y}, \Phi, R_{f}, R_{r}, \delta_{f}, v$, and the forces of the brake on the left and right hand side $F_{b l}, F_{b r}$.

As the lateral acceleration increases in the maneuver it generates a moment to rollover the vehicle. The normalized load transfers also increase. The lateral acceleration in both the passive and the active case is identical if the normalized load transfers do not reach the critical value, but after the critical value is exceeded the control algorithm is activated and the active brake system reduces the lateral acceleration. The second driving maneuver is more critical than the first one. The active brake is only activated in the second maneuver at $2.6 \mathrm{sec}$ and decreases the lateral acceleration and so the normalized load transfers. At the time when the brake control is activated the rear-left wheel is braked to avoid the rollover of the vehicle. Approximately $57 k N$ maximal control force is required for the rear-left wheel during this maneuver. In the passive case the forward velocity is constant during the cornering maneuver because it is assumed the driver is not pushing on the brake pedal. In case of the active brake control the forward velocity is not constant because the brake force provided by the active control decelerates the vehicle. In the passive case the normalized load transfers are above the value \pm 1 , which means that the lateral force on one of the curve inner side wheels becomes zero.

In the second example, the active brake is compared with the active anti-roll bars. Here, there is not any prediction procedure in the operation of the active brake. Figure 4 shows solid (active brake) and dashed (active anti-roll bars) lines the time responses to the double lane change steering input. The active anti-roll bars do not influence directly the yaw motion of the vehicle
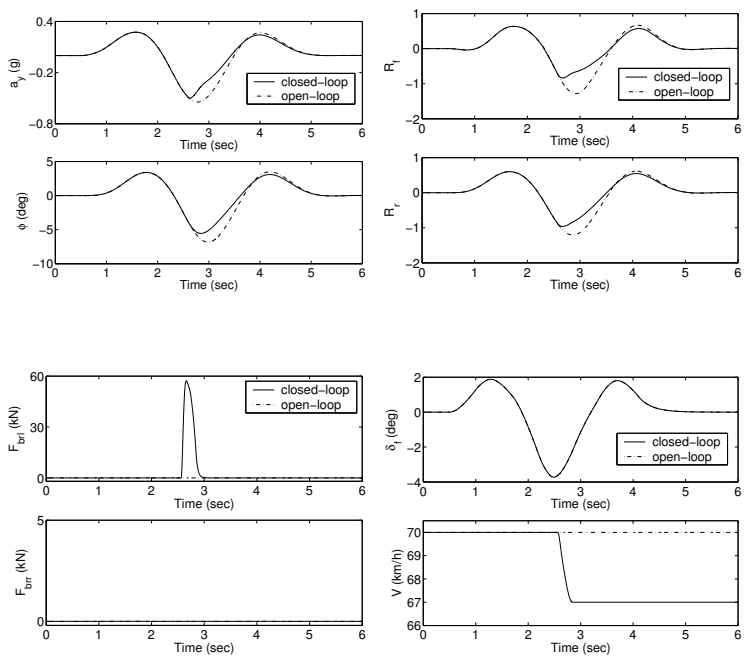

Fig. 3. Time responses to double lane change steering input
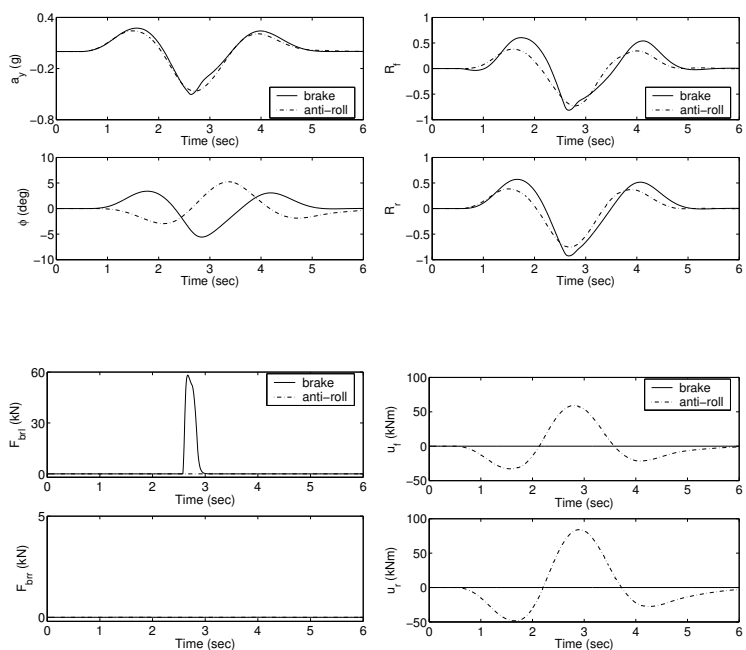

Fig. 4. Time responses to double lane change steering input

while the brake control do. Thus, the active antiroll bars operate all the time and they generate a stabilizing moment to balance the overturning moment caused by lateral acceleration. Approximately $59 \mathrm{kNm}$ maximal moment is required between the sprung and unsprung mass at the front side and $84 \mathrm{kNm}$ maximal moment is required at the rear side during this maneuver. The disadvantage of the active anti-roll bars is that the maximum stabilizing moment is limited physically by the relative roll angle between the body and the axle.

In the third example, the active brake used in a prediction procedure is compared with the active brake, in which there is not any prediction procedure. The vehicle dynamics is illustrated for a zoomed interval from $1.5 \mathrm{sec}$ to $3.5 \mathrm{sec}$ in Figure 5. The figure illustrates that the magnitude of 

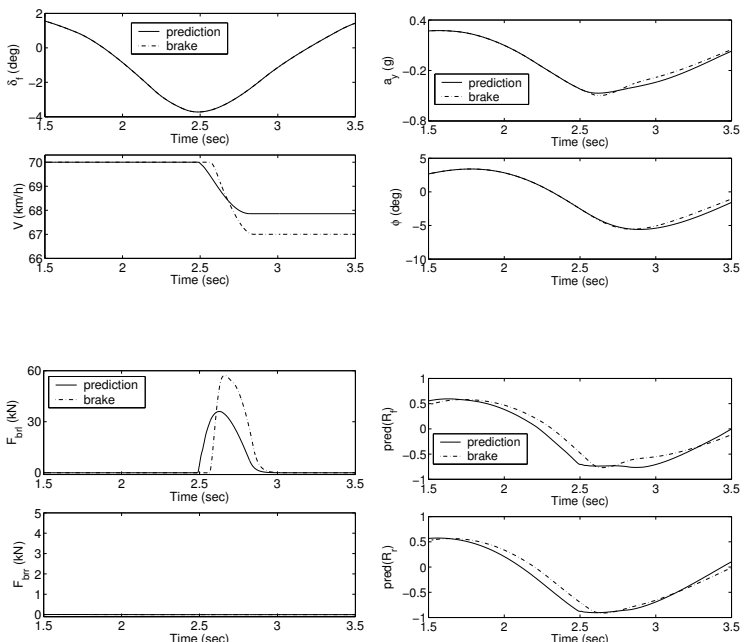

Fig. 5. Time responses to double lane change steering input

the braking process is smaller and the duration is shorter in the case of the active brake which uses a prediction procedure than the brake without prediction. The brake control is activated earlier in the predicted case, i.e. at $2.5 \mathrm{sec}$, than in the brake without prediction. The maximal control force required is approximately $36 k N$ for the rearleft wheel during this maneuver. The control force required is smaller approximately by $40 \%$ in the predicted case than in the traditional case. The figures also show the dependable prediction of the normalized load transfers through the observer design and the prediction algorithm using a short time interval.

\section{CONCLUSION}

The aim of this paper is to improve the roll stability of heavy vehicles by applying an active brake system based on the LPV modelling and control design. To enhance the performance of the active brake, this mechanism is extended with a prediction procedure, in which the critical values are predicted in advance. In the LPV modelling the forward velocity and the normalized lateral load transfer at the rear side are chosen as scheduling parameters. The control design based on the LPV method results in a compensator which meets the performance specifications and takes the model uncertainties into consideration. The simulation example has shown that smaller and shorter braking force is is required in the predicted case than without prediction. The efficiency of the braking is improved for brakes extended with prediction.

Acknowledgement: This work was supported by the Hungarian National Office for Research and Technology through the project "Advanced Vehicles and Vehicle Control Knowledge Center"
(OMFB-01418/2004) and the Hungarian National Science Foundation (OTKA) under the grant $T-$ 048482 which are gratefully acknowledged. Dr Gaspar was supported by the Janos Bolyai Research Scholarship of the Hungarian Academy of Sciences.

\section{REFERENCES}

Ackermann, J. and D. Odenthal (1999). Damping of vehicle roll dynamics by gain scheduled active steering. Proc. European Control Conference.

Chen, B. and H. Peng (2001). Differentialbraking-based rollover prevention for sport utility vehicles with human-in-the-loop evaluations. Vehicle System Dynamics 36, 359389.

Gahinet, P., P. Apkarian and M. Chilali (1996). Affine parameter dependent lyapunov functions and real parameter uncertainity. IEEE Transactions on Automat. Contr. 41, 436442.

Gaspar, P., I. Szaszi and J. Bokor (2004). The design of a combined control structure to prevent the rollover of heavy vehicles. European Journal of Control 10(2), 1-15.

Gaspar, P., I. Szaszi and J. Bokor (2005). Reconfigurable control structure to prevent the rollover of heavy vehicles. Control Engineering Practice 13(6), 699-711.

Kim, H.J. and Y.P. Park (2004). Investigation of robust roll motion control considering varying speed and actuator dynamics. Mechatronics 14, 35-54.

Lin, R.C., D. Cebon and D.J. Cole (1996). Optimal roll control of a single-unit lorry. Proc. IMechE, Journal of Automobile Engineering pp. $44-55$.

Ljung, L. (1987). System identification: Theory for the user. Prentice-Hall, Inc., Englewood Cliffs, New Jersey.

Mammar, S. and D. Koenig (2002). Vehicle handling improvement by active steering. Vehicle System Dynamics 38, 211-242.

Palkovics, L., A. Semsey and E. Gerum (1999). Roll-over prevention system for commercial vehicles. Vehicle System Dynamics 32, 285297.

Sampson, D.J.M. and D. Cebon (2003). Active roll control of single unit heavy road vehicles. Vehicle System Dynamics 40, 229-270.

Wu, F., X. H. Yang, A. Packard and G. Becker (1996). Induced $\mathbf{l}^{2}$ norm control for lpv systems with bounded parameter variation rates. International Journal of Nonlinear and Robust Control 6, 983-998. 